\title{
Pressure distribution in a quasi-hydrostatic pressure medium: A finite element analysis
}

\section{Citation}

Tempere, Jacques, and Isaac F. Silvera. 2011. "Pressure Distribution in a Quasi-Hydrostatic Pressure Medium: A Finite Element Analysis." Journal of Applied Physics 110 (11): 113523. doi:10.1063/1.3665194.

\section{Published Version}

doi:10.1063/1.3665194

\section{Permanent link}

http://nrs.harvard.edu/urn-3:HUL.InstRepos:29374808

\section{Terms of Use}

This article was downloaded from Harvard University's DASH repository, and is made available under the terms and conditions applicable to Other Posted Material, as set forth at http:// nrs.harvard.edu/urn-3:HUL.InstRepos:dash.current.terms-of-use\#LAA

\section{Share Your Story}

The Harvard community has made this article openly available.

Please share how this access benefits you. Submit a story.

Accessibility 


\title{
Pressure distribution in a quasi-hydrostatic pressure medium: A finite element analysis
}

\author{
Jacques Tempere ${ }^{1,2}$ and Isaac F. Silvera ${ }^{2, a)}$ \\ ${ }^{1}$ TQC, Universiteit Antwerpen, Groenenborgerlaan 171, B-2020 Antwerp, Belgium \\ ${ }^{2}$ Lyman Laboratory of Physics, Harvard University, Cambridge, Massachusetts 02138, USA
}

(Received 26 February 2011; accepted 27 October 2011; published online 12 December 2011)

\begin{abstract}
The highest quality pressures on samples in a diamond anvil cell (DAC) at high pressures are produced using quasi-hydrostatic pressurization media such as helium or hydrogen. In this paper we carry out a finite element analysis of pressure distributions in a DAC using helium and non-hydrostatic argon pressurization media. We find that samples and ruby chips are at substantially higher pressures than the pressurization media, although this is sharply reduced by using helium, which has a low yield strength for the shear modulus. The deviations in pressure of the different samples (and ruby) from the pressurization media differ and depend on their elastic constants. Our observations may account for the distribution of pressures in metallic markers found in a recent calibration of the ruby scale to high pressures. (C) 2011 American Institute of Physics.
\end{abstract}

[doi:10.1063/1.3665194]

\section{INTRODUCTION}

Pressures in the megabar range are achieved in diamond anvil cells (DACs). Ideally the sample being studied would be pressurized by a hydrostatic pressure medium. However, there are no known materials that exist in the liquid phase at these extreme pressures and ambient temperatures, so solids such as helium or hydrogen are used as quasi-hydrostatic pressure media. These media are quite soft at low pressures and have low yield strengths; experience has demonstrated that helium is the best available medium and shows little characteristic evidence of deviatoric or non-hydrostatic stress in high-pressure measurements. ${ }^{1-3}$ Even though the structures are generally in the hcp phase and the stress-strain conditions are quite homogeneous, ${ }^{4}$ the important parameter for a quasihomogeneous behavior is the yield strength.

Pressures in a DAC are determined either from the laser excited ruby fluorescence or by the equation of state of a metal marker measured by x-ray diffraction; in addition to these standard techniques of measuring pressure, in the past decade the shift of the Raman active phonon in diamond has been used as a secondary pressure gauge. In the ruby technique a ruby particle, typically a few micrometers in linear dimensions, is embedded in the pressure medium and its ruby fluorescence spectrum is measured; the pressure is determined from the wavelength of the ruby $\mathrm{R} 1$ line which shifts with pressure and this shift is calibrated. In the marker technique a piece of metal, also a few micrometers in linear dimension, is embedded in the pressure medium and is X-rayed to determine the lattice parameter or the volume. The pressure is determined from the known P-V equation of state (EOS). It is generally assumed that the pressure medium, the marker or sample, and the ruby are all at the same pressure. Ruby is cali-

\footnotetext{
a) Author to whom correspondence should be addressed. Electronic mail: silvera@physics.harvard.edu.
}

brated against metals with known EOSs by measuring the ruby shift and the x-ray diffraction of metal markers, both in the same quasi-hydrostatic pressure medium. In the diamond technique, originally the shift of the diamond phonon was calibrated in a plate of diamond embedded in a pressurization medium, ${ }^{5,6}$ however this was limited, as at higher pressures the diamond anvils and the diamond plate would bridge. Boppart, van Stratten, and Silvera ${ }^{6}$ proposed using the high frequency wing extended to zero amplitude to determine and calibrate the pressure and this has been done by a number of researchers. $^{7-11}$ More recently Dubrovinskaia et al. ${ }^{12}$ have produced micrometer sized diamond markers embedded in the pressurization medium and provided a calibration to megabar pressures. Thus, we also include diamond markers in our study.

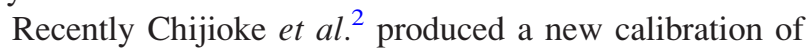
ruby against a set of 6 metal markers. The EOSs of these markers were derived from shock-wave data and believed to be accurate to within $1 \%$. A new ruby calibration called $\mathrm{CNSS05,}{ }^{2}$ with significant differences from the older generally used so-called quasi-hydrostatic scale of Mao, $\mathrm{Xu}$, and Bell, ${ }^{13}$ was produced. The deviations of the pressure of each metal marker from the new scale were plotted against the pressure of this new scale. If there were no systematic errors or shifts, then the data plotted in this way should be randomly distributed around the pressure axis. However, this plot was found to give a "fan" of values as shown in Fig. 1. These deviations were larger than the estimated errors for the EOSs of the markers. A possible explanation is that the pressures in the ruby and markers were different from the pressures in the quasi-hydrostatic pressure media. This is certainly possible because the pressure media were solids and not hydrostatic.

In this paper we carry out a finite element analysis (FEA) of the pressure distributions in the pressurization media, the markers, and the ruby, in a DAC as a function of pressure. We find that the pressure is not uniform in the media, nor are 


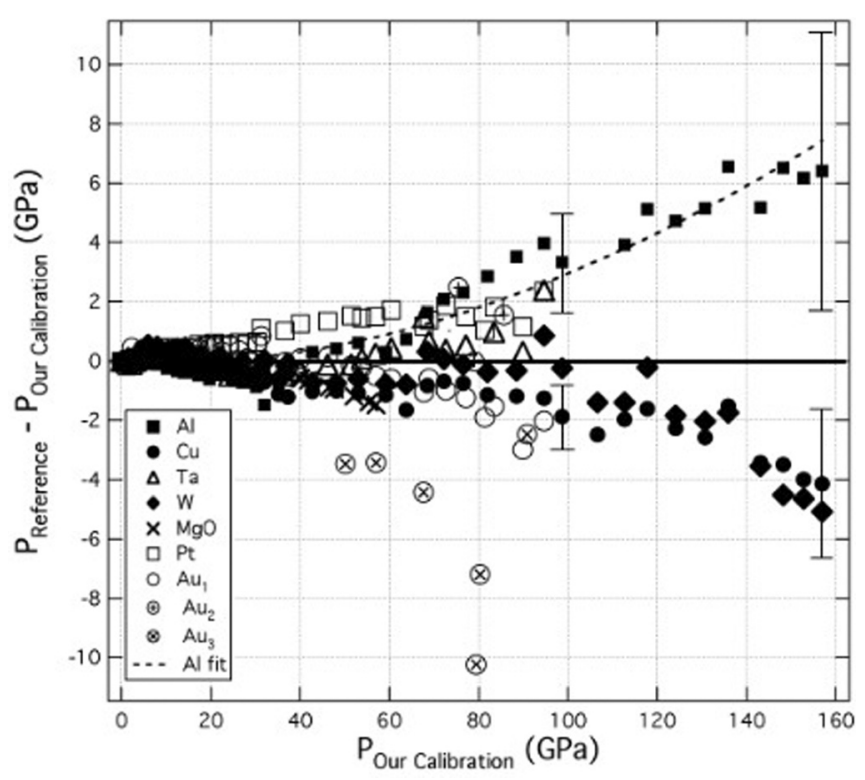

FIG. 1. The pressure in metal markers vs the deviation from the calibrated pressure of ruby forms a "fan" of curves for different metals (from Ref. 2). The original marker data is from Ref. 1. Information on the markers is that they were placed at the center of the cell and were $4 \mu \mathrm{m}$ in maximum dimension. A $4 \mu \mathrm{m}$ diameter ruby ball was adjacent to the metal grains.

the average pressures in the ruby chips or markers the average pressure of the media. Fortunately, these deviations are small (in helium) so that pressures as measured will be reasonably representative of the pressure of an embedded sample. However, they are large enough to possibly explain the "fan" of deviations discussed above.

\section{ANALYSIS PROCEDURE}

We perform a finite element analysis (FEA) using the commercial program, COMSOL MULTIPHYSICS. The geometry that we have used corresponds to that of a DAC, as shown in Fig. 2. This consists of two opposing diamonds, separated by a rhenium gasket with the hole in the gasket filled with the pressurization medium, either helium or argon. Embedded in the medium is a marker disk at the center of symmetry, with height $4 \mu \mathrm{m}$ and diameter $4 \mu \mathrm{m}$, and $1 \mu \mathrm{m}$ above the bottom diamond. The marker can be replaced by ruby or an embedded sample, using appropriate elastic constants. The gasket has an initial (zero-pressure) thickness of $30 \mu \mathrm{m}$ and a hole of $120 \mu \mathrm{m}$ diameter. Helium is known to provide quasihydrostatic pressures, while argon becomes non-hydrostatic at a pressure of the order of $12 \mathrm{GPa}^{6}$ This might account for the differences with the early calibration in argon. ${ }^{13}$ The DAC and sample are at room temperature and we only consider the equilibrium static pressure distributions, the temperature being uniform. In this analysis we shall call the contents of the pressure medium in the gasket the "sample" (the marker also plays the part of a real sample that might be under study). Opposing forces are applied to the two diamonds to produce a desired pressure in the medium. The local pressure is defined as the (trace of the stress tensor)/ 3 (an output of the FEA) and average pressure as the pressure averaged over a designated volume such as the marker or the pressurization medium. The pressure can be measured at any point.

Although the cell geometry should produce a uniaxial stress on a solid sample, if the pressurization medium is liquid, then it will produce a hydrostatic pressure on embedded materials. If the shear forces in the medium yield at a low value then the medium will produce a quasi-hydrostatic pressure. Most high-pressure studies treat the pressure in a quasihydrostatic medium to be hydrostatic. Here we study the deviations from hydrostaticity. In our analysis we first used a three-dimensional geometry with cylindrical symmetry, as shown in Fig. 2. This geometry is very demanding on computer memory and time. We found essentially the same results using a two-dimensional model that assumes the geometry shown in Fig. 2 is infinite, in and out of the page. When we studied markers or ruby that were not located at the center of the cell, we used the 2D model.

The FEA structural mechanics module solves the force equations to give an equilibrium state solution. The FEA meshes can be non-uniform with increased density in regions of interest or where gradients are large. Meshes substantially smaller than the smallest elements under analysis were implemented and the net could be adjusted until the solution converged to a stable result. The general equilibrium equation that is solved is,

$$
\frac{E}{2(1+v)} \Delta \mathbf{u}+\frac{E}{2(1+v)(1-2 v)} \nabla(\nabla \cdot \mathbf{u})+\mathbf{f}=0 .
$$

Here $\mathbf{u}$ is a displacement vector, $E$ is Young's modulus, $v$ is Poisson's ratio, and f represents body forces. The boundary conditions are that the displacements are continuous from element to element and that there is a uniaxial load on the top and bottom diamonds. Thus, at an interface, for example, that of the diamond and the medium, the displacements are continuous but the elastic constants are not. As a result, the stresses will not be continuous when moving from one material into another. The input for the FEA program consists of the geometry, the elastic constants of the different materials, and the load. We list the elastic constants used for the different materials in Table I. It is sometimes easier to find data for the bulk modulus $K$ and the shear modulus $G$ in the literature, and one can transpose this data to Young's modulus

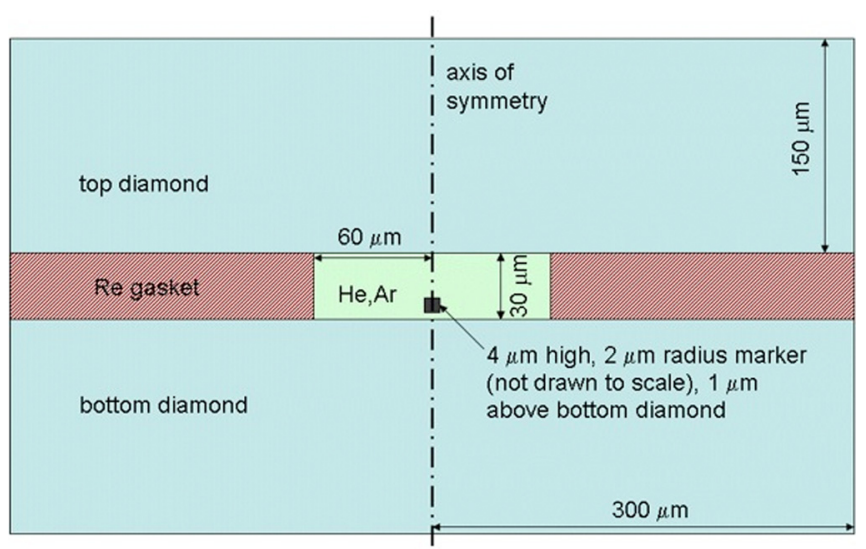

FIG. 2. (Color online) The geometry used in the finite element analysis. 
TABLE I. Material parameters used in the numerical calculations. Simple fitting formulae are used to model the pressure dependence of Young's modulus $E$ and the density $\rho$; pressure in these formulae is in units of GPa. The Poisson ratio $v$ is taken independent of pressure, except in the case of argon and helium. In the region of 20-150 GPa these fitting formulae give results within 1\% accuracy with the experimental results reported in the sources. For the fields left blank in the table, we used slightly different phenomenological formulae to obtain a good fit:

\begin{tabular}{|c|c|c|c|c|c|c|c|}
\hline \multirow[b]{2}{*}{ Material } & \multicolumn{3}{|c|}{$E=\mathrm{a}+\mathrm{bP}-\mathrm{cP}^{2}(\mathrm{GPa})$} & \multirow[b]{2}{*}{$v$} & \multicolumn{3}{|c|}{$\rho=\mathrm{a} \exp [\mathrm{b} \log (1+\mathrm{cP})]\left(\mathrm{kg} / \mathrm{m}^{3}\right)$} \\
\hline & $\mathrm{a}$ & $\mathrm{b}$ & $\mathrm{c} \times 10^{-2}$ & & $\mathrm{a}$ & $\mathrm{b}$ & $c \times 10^{-2}$ \\
\hline $\mathrm{Ta}^{\mathrm{a}}$ & 178.156 & 2.9677 & 0.180745 & 0.35 & 16650 & 0.345329 & 1.37456 \\
\hline $\mathrm{Al}^{\mathrm{a}}$ & 77.0383 & 3.60459 & 0.152976 & 0.34 & 2700 & 0.285178 & 4.05953 \\
\hline$\omega-\mathrm{Ti}^{\mathrm{a}}$ & 151.577 & 6.34567 & 0.427608 & 0.32 & 4507 & 0.194957 & 3.31316 \\
\hline $\mathrm{Cu}^{\mathrm{a}}$ & 151.387 & 6.89044 & 0.60759 & 0.34 & 8920 & 0.151127 & 4.00197 \\
\hline $\mathrm{W}^{\mathrm{a}}$ & 385.028 & 4.93536 & 0.205837 & 0.29 & 19250 & 0.278534 & 1.13642 \\
\hline $\mathrm{Pt}^{\mathrm{a}}$ & 207.485 & 3.48016 & 0.15955 & 0.38 & 21090 & 0.227772 & 1.45276 \\
\hline $\mathrm{Au}^{\mathrm{a}}$ & 60.7388 & 1.86663 & 0.10404 & 0.44 & 19300 & 0.212413 & 2.59852 \\
\hline $\operatorname{Re}^{\mathrm{b}}$ & 460.274 & 6.42074 & 0.29407 & 0.29 & 21020 & 0.216713 & 1.21792 \\
\hline hcp $\mathrm{Fe}^{\mathrm{c}}$ & 163.782 & 4.54139 & 0.223289 & 0.34 & 7874 & 0.230051 & 2.40679 \\
\hline Ruby $^{\mathrm{d}}$ & 438.884 & 6.52117 & 0.280372 & 0.22 & 3970 & 0.281859 & 1.30528 \\
\hline Diamond $^{\mathrm{e}}$ & 1156.7 & 7.44773 & 0.387805 & 0.069 & 3515 & 0.387433 & 0.564832 \\
\hline $\operatorname{Ar}^{\mathrm{f}}$ & 3.02781 & 4.49343 & 0.738171 & & 2000 & 0.286727 & 28.7437 \\
\hline $\mathrm{He}$ & 6.09109 & 2.87352 & 0.0 & & & & \\
\hline
\end{tabular}

$\nu_{\mathrm{Ar}}=(27.9001+7.45787 \times \mathrm{P}) /(66.6158+24.3814 \times \mathrm{P})$

$\nu_{\mathrm{He}}=(28.8637+5.85982 \times \mathrm{P}) /(78.6849+18.1488 \times \mathrm{P})$

$\rho_{\mathrm{He}}=988.1 \times \exp [0.344889 \times \log (0.130387+0.0668933 \times \mathrm{P})]$

${ }^{\mathrm{a}}$ Ref. 17.

${ }^{\mathrm{b}}$ Ref. 18.

${ }^{\mathrm{c}}$ Refs. 19,20 .

${ }^{\mathrm{d}}$ Ref. 21.

${ }^{\text {e}}$ Refs. 22,23. Note that these values are for diamond under hydrostatic pressure, not under uniaxial pressure, so these are the values used for a diamond chip in the cell rather than the anvil diamonds. For the anvils, as long as the diamond has much higher bulk modulus and a small Poisson ratio, the results are qualitatively independent of the precise values. For the anvils we use $E=1200 \mathrm{GPa}, \nu=0.1$ and $\rho=3500 \mathrm{~kg} / \mathrm{m}^{3}$.

${ }^{\mathrm{f}}$ Ref. 24. These results are up to $70 \mathrm{GPa}$.

and the Poisson ratio using the standard relations for homogeneous isotropic materials:

$$
E=\frac{9 K G}{3 K+G} \quad \text { and } \quad v=\frac{3 K-2 G}{6 K+2 G}
$$

One of the challenges of this study was to find elastic data for the properties of materials under study as a function of hydrostatic pressure. In order to achieve realistic results we have gathered pressure-dependent data for the many materials in this problem, fitted the data to appropriate formulae, and extrapolated them over some limited ranges when necessary. Small errors may accrue due to these extrapolations, but should not create a serious problem; moreover, we are interested in trends. These functional forms and their source references are given in Table I. The values of the material parameters at a given pressure were input at the average pressure of the element (marker, ruby, or medium). The pressure dependence of the elastic moduli essentially introduces nonlinearity in Eq. (1).

The computational procedure that was followed can be split into several steps. First, a load is applied to the diamonds, and initial guesses for the pressure are used to obtain the elastic parameters of the different materials needed as input for the program. Next, Eq. (1) is solved numerically, applying the boundary conditions. Then, the average pressure is determined in the marker and the medium. Finally, the elastic parameters of the marker and the medium are adapted to the values corresponding to the determined average pressure. This procedure is iterated until the pressures have converged. Although the pressure dependence of the elastic constants of the marker (or ruby) and the medium are important, this is not the case for the diamond anvils and the rhenium: As long as their bulk modulus strongly exceeds that of the pressure medium, the precise value is not important.

If just the unconstrained elastic constants of a pressurization medium such as helium are used, then substantial pressure variations are found within the pressurization medium and the embedded samples. For our study here we are interested in the yielding of the (softer) pressurization media, in particular helium, for which there is very little data on its yield strength as a function of pressure. Because general theories of yielding predict that the strength of a material is proportional to its shear modulus, ${ }^{14}$ we have used the following approach. To model the pressure-dependent yield strength we have yielded the shear modulus $\mathrm{G}_{\text {yield }}(\mathrm{P})=G(\mathrm{P}) / 10$ for helium. This effectively reduces the pressure gradients to values comparable to what is observed in experiments. ${ }^{15}$ For argon we have not put in a yield strength. To implement the yield into the analysis, we set $\mathrm{G}_{\text {yield }}(\mathrm{P})=G(\mathrm{P}) / 10$ and then solved for $E$ and $v$ using Eq. (2). This last step in the procedure is carried out because the FEA program input is Young's modulus and the Poisson ratio, not the shear modulus.

In a DAC under load, the gasket yields or undergoes plastic deformation. In this study we did not put in a low yield strength of the rhenium gasket, as this added complication 
and would not seriously affect the results at the boundary between the pressure medium and the gasket. Although the gasket and other materials deform as a function of pressure, the gasket remains quite thick at high pressures. The pressure in the gasket at the interface with the pressure medium rises to a value that is high relative to that of the medium because of the strength of the gasket.

\section{RESULTS OF THE ANALYSIS}

\section{A. Pressure difference between medium and marker}

Already in the simplest simulations it was clear that the average hydrostatic pressure in the marker can differ substantially from the average hydrostatic component of the pressure in the medium. Figure 3 illustrates the distribution in the pressure throughout the cell and marker. The variation of pressure is largest when we do not model a yield strength in helium, or when we use argon. Placing a marker in the medium also locally alters the hydrostatic pressure in the cell. For markers with a Poisson ratio smaller than that of the medium, the presence of the marker relieves strain in the medium above and below the marker, whereas it increases stress to the sides of the marker. For markers with a Poisson ratio

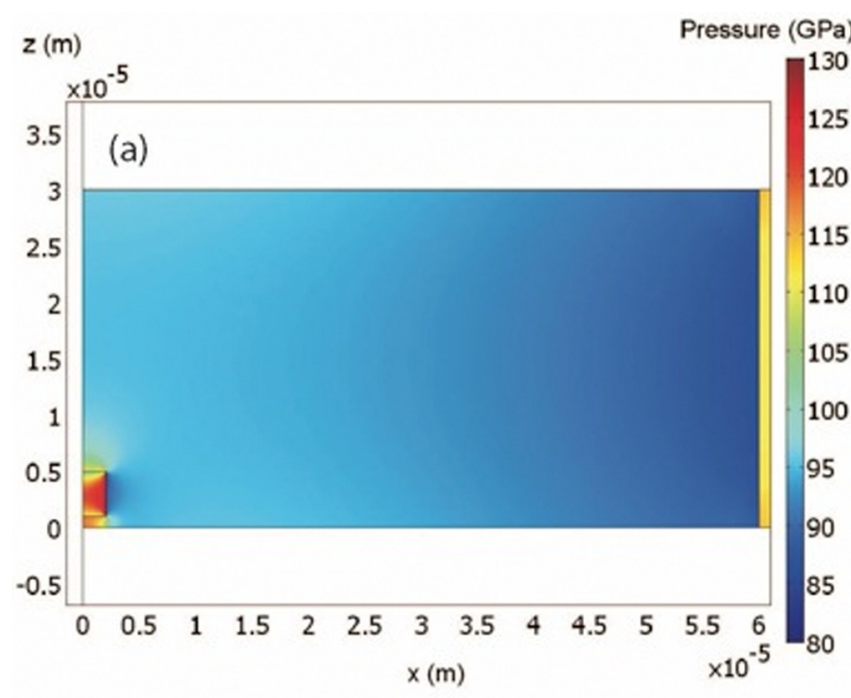

(b)

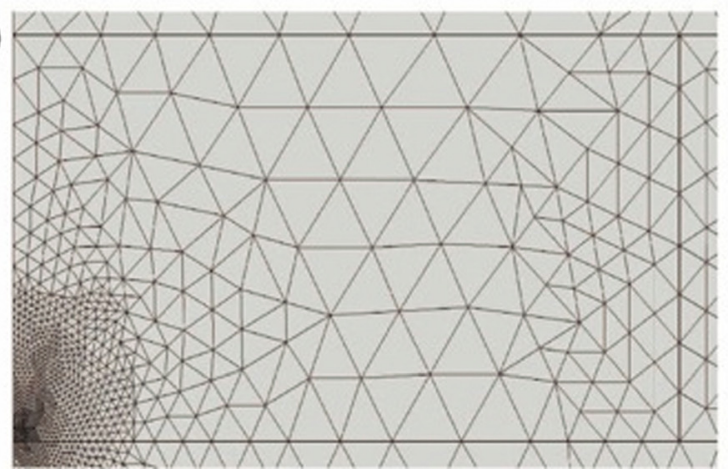

FIG. 3. (Color online) (a) Pressure distribution in the cell. This extreme example, an aluminum marker in helium where no yielding of the shear modulus has been taken into account, clearly shows that the pressure is higher in the marker (110-120 GPa) than the medium (100 GPa), and that the medium is locally affected. (b) A typical mesh used for the analysis. Near the marker, the mesh is refined, to be adapted to the small size of the marker with respect to the cell. larger than the medium, this is reversed. In both cases, the average pressure in the marker is higher than that of the medium. This is linked to the boundary condition of continuity of the strain field in Eq. (1): As one passes from the medium into the marker, the larger Young's modulus of the marker leads to a larger stress for a similar strain. An example for the pressure measured in ruby versus the pressure in the medium is shown in Fig. 4.

\section{B. Pressure differences between different metals}

The pressure difference between marker and medium is due to the fact, stated earlier, that the strain varies continuously whereas the elastic constants change abruptly from medium to marker. The various marker materials are characterized by different elastic constants, and this will lead to different average hydrostatic pressures in the different metals, even when they are placed in the same spot and subjected to the same load. In Fig. 5 we show the pressure in the various materials (metallic markers and ruby) as a function of the pressure in helium and argon pressurization media (with no relaxation of the shear) averaged over the values in all of the metals, ruby, and diamond. Such an average pressure might be used in a calibration using many different marker materials. However, it is clear that when one uses only one marker, say aluminum, a very different (10\%) calibration is obtained than when one would use, say, platinum.

The magnitude of the fan of curves in Fig. 5 is larger than the magnitude of the fan observed experimentally. To achieve a spread of marker pressures comparable to what is observed in experiment, the helium shear modulus is yielded, shown in Fig. 6. Even though the calculated pressures show the same systematic trends, there is no one-to-one correspondence in the order of the materials in the fan. For example,

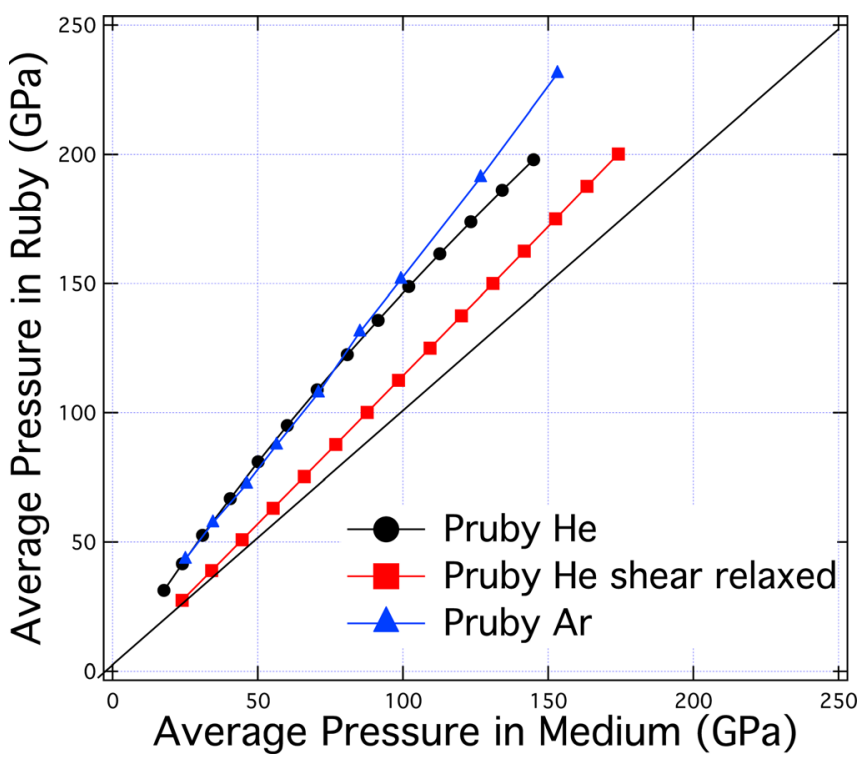

FIG. 4. (Color online) A plot of the average pressure in the ruby chip vs the average pressure in the pressurization medium. Plots are shown for ruby in argon, in helium, and in helium with the shear modulus relaxed to $10 \%$ of its value determined from the elastic constants. In our analysis here, the ruby was placed at the center of the cell where the pressure in the medium is higher than the average pressure of the medium. 

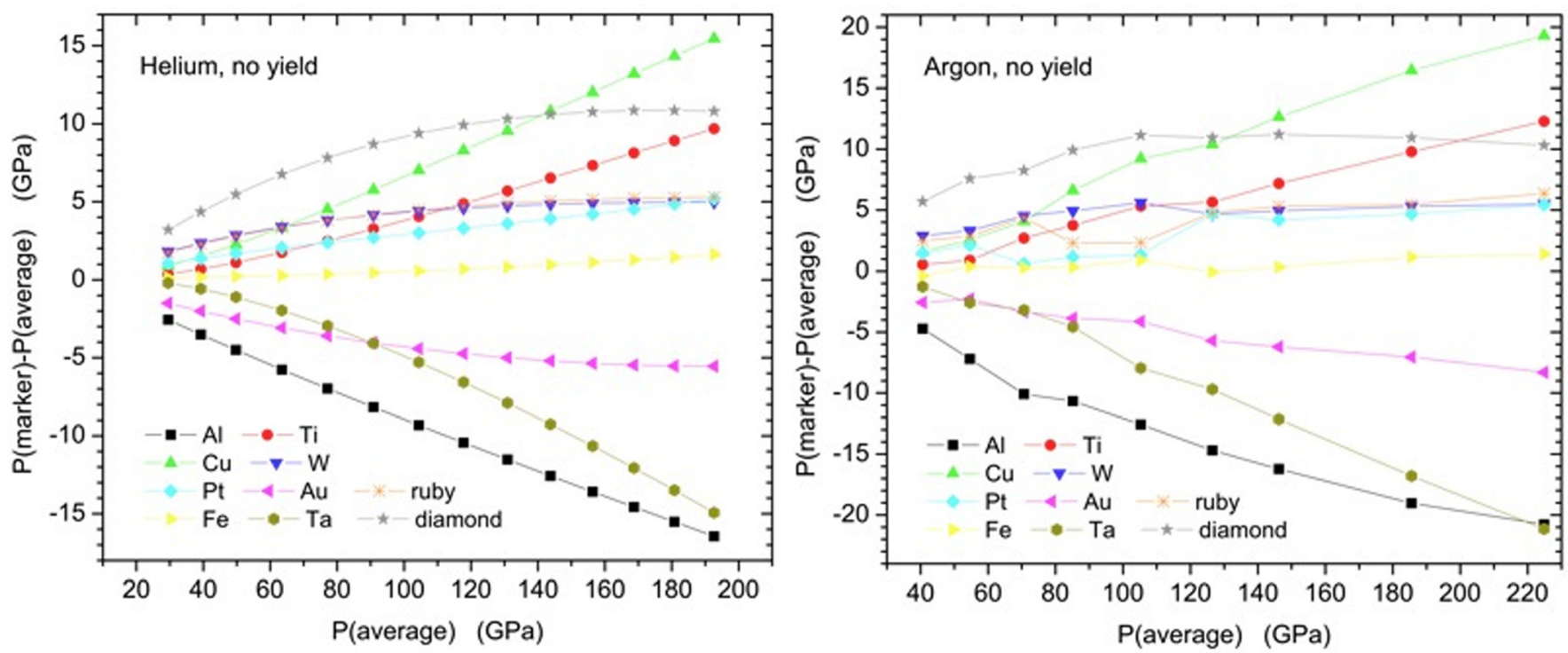

FIG. 5. (Color online) The pressure deviations in various markers, including ruby and diamond, as a function of the pressure averaged over all the different marker materials.

in the experimental fan shown in Fig. 1, Al lies higher than $\mathrm{Pt}$, whereas in our simulation the reverse is true. This indicates that there can be other systematic errors affecting the calibrations, on top of the systematics due to elastic constant differences described in this paragraph. In Sec. III C, we identify two other sources of systematic errors: marker shape and marker position in the cell. This list is, of course, not necessarily comprehensive: There can be other systematic errors in, for example, the shock-wave reduced isotherms used for the calibrations.

\section{Pressure differences due to marker shape}

Not only the marker material matters, but also (to a lesser extent) the shape of the marker. Figure 7 shows the pressure in various metals and in ruby when the shape of the material is changed from a cylinder (square cross-section of $4 \mu \mathrm{m}$ high and $4 \mu \mathrm{m}$ diameter) to a sphere (with $4 \mu \mathrm{m}$ diame-

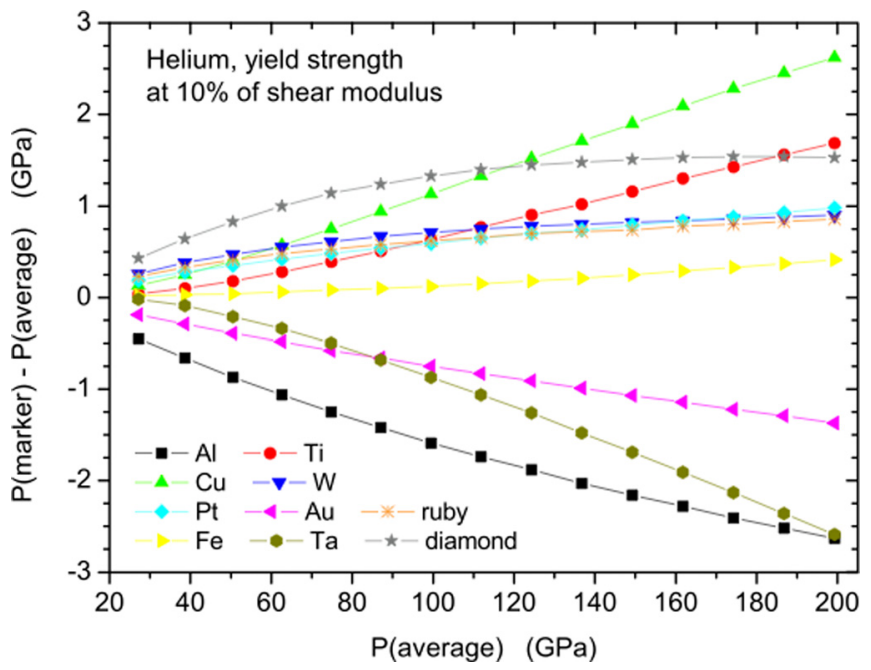

FIG. 6. (Color online) Same as Fig. 5, but the shear modulus in the helium pressurization medium is relaxed to $10 \%$. ter). Filleting the corners continuously changes the shape. The different materials all react in the same manner, so that one would obtain the same fan of pressures with spherical markers or with cylindrical markers. The gold marker is less sensitive than the other materials to the shape of the grain, possibly because of the large value of the Poisson constant (0.44) for this material. Note that when one compares cylindrical Al markers with cylindrical Pt markers, the Pt curve in the fan will always be higher than the Al curve. But when one compares cylindrical $\mathrm{Al}$ markers with spherical $\mathrm{Pt}$ grains, then the curve of $\mathrm{Al}$ will lie higher than that of $\mathrm{Pt}$ in the fan.

\section{Pressure gradients in the cell}

If one puts a load on a solid gasket without a hole, then the pressure is peaked at the center and falls off to ambient

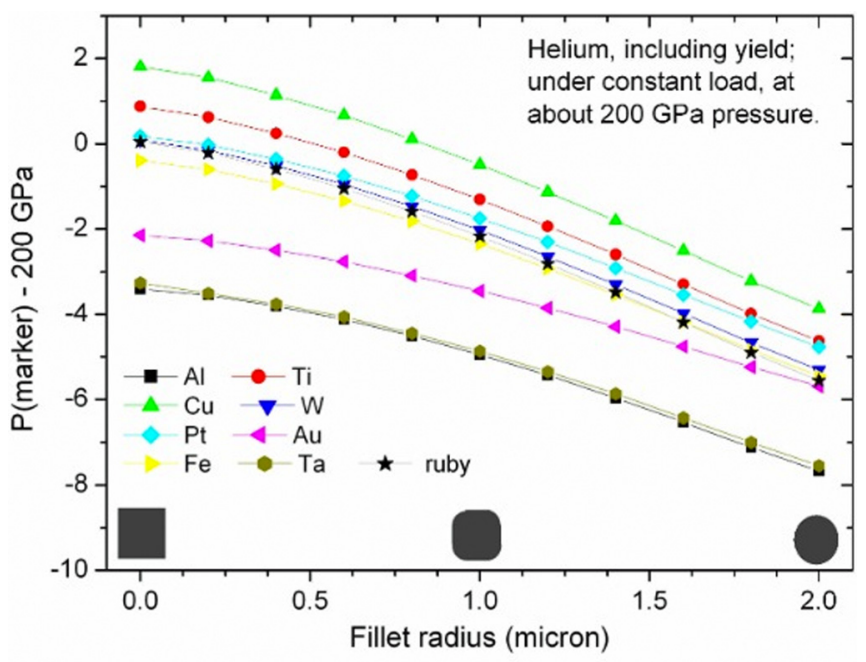

FIG. 7. (Color online) The pressure in the markers is shown as a function of marker shape (varying continuously from cylindrical to spherical) for different marker materials. The load on the diamonds and the position of the marker is kept constant. 


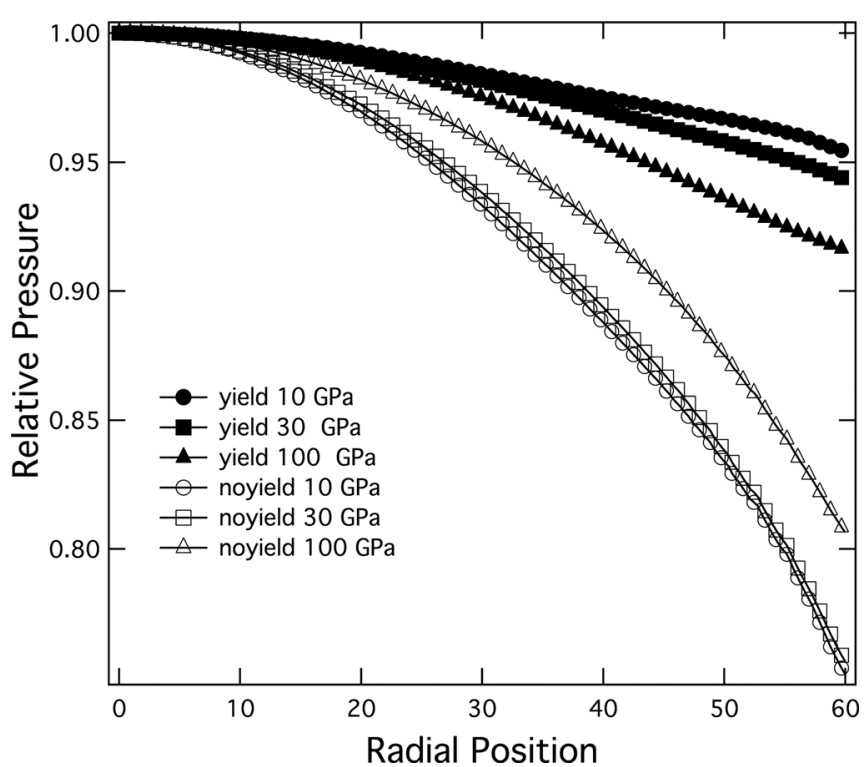

FIG. 8. The radial pressure variation in a helium pressurization medium for the case of helium with no yield or with yield in the shear modulus. These results will depend on the strength of the gasket material.

at larger radius. This behavior is easily found from the simple equation,

$$
h \frac{d \sigma_{r}}{d r}+\sigma_{0}=0
$$

where $h$ is the gasket thickness, $\sigma_{r}$ is the radial stress or pressure in this case, and $\sigma_{0}$ is the compressive yield stress of the gasket. ${ }^{16}$ The pressure in the medium (measured in the FEA without a marker or ruby, which locally disturbs the surrounding pressure) also shows a weak radial fall-off of pressure, as is the case for a solid gasket, but strongly reduced. In Fig. 8 we show the local radial pressure for both helium with no yield and with yield in the shear modulus.

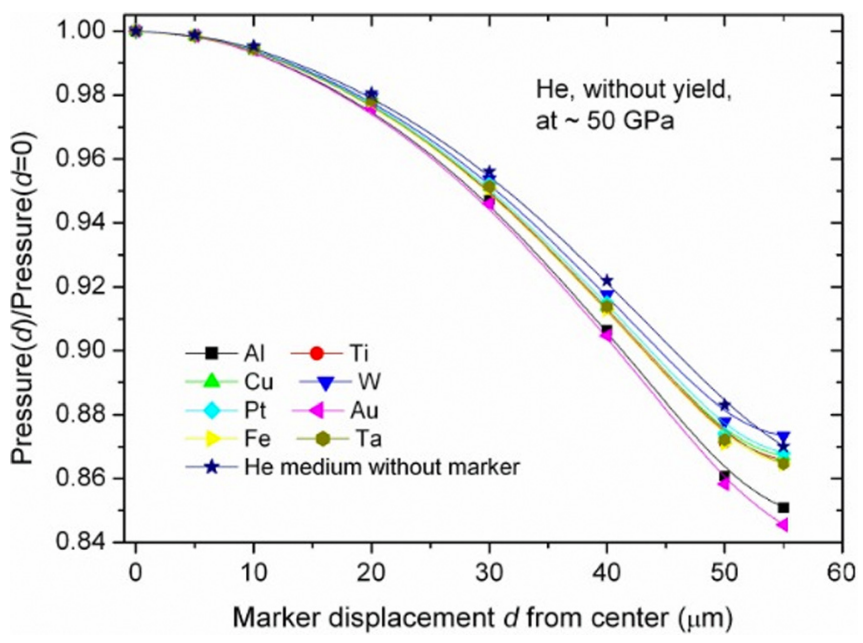

FIG. 9. (Color online) The average pressure of a displaced marker, relative to the pressure of that same marker placed at the center of the gasket. Replacing the marker by helium (the medium) we see that the relative pressure drop related to displacing the marker out of the center corresponds to the pressure gradient in the (unperturbed) medium in the cell.
Depending on the position of the marker in the cell, the local hydrostatic pressure squeezing the marker will be different. Figure 9 shows the hydrostatic pressure in the markers as a function of the distance from the center of the gasket hole. The relative reduction in pressure is the same for all the materials studied, and follows the relative reduction in pressure in the medium. Note that when working with relatively nonhydrostatic media the pressure gradients in the cell can be important. In order to investigate the effects of displacing the marker away from the center of the cell, we did not do a separate full-3D simulation, but rather relied on a plane-strain $2 \mathrm{D}$ calculation. A comparison of these results with the full-3D case is not straightforward, because the shape of the marker influences the pressure as well; and with a given 2D profile, different 3D shapes can be constructed. Our aim here is to indicate that the displacement of any given grain from the center has an effect on the determined pressure comparable to the effects of shape and size of the grain.

\section{CONCLUSION}

From these figures it is clear that the conditions in the helium loaded DAC are far from hydrostatic, but the low yield shear strength of the helium medium is important for reducing the non-hydrostatic stress distributions. From Figs. 4 and 5, 6, and 8 we see that the pressures measured in the different markers are different from the average pressure of the medium and the ruby; that is, if we plot the marker pressures as a function of the medium or ruby pressures, we get a "fan" of values, similar to the experimentally observed fan represented in Fig. 1, used in determining the ruby pressure scale. Systematic pressure differences arise from several sources: differences in elastic constants, in shape, or in position in the cell. Our results can easily account for the differences found for gold pressures determined in helium or xenon (less quasi-hydrostatic then helium). A nice compensating feature for the calibrations is that both the ruby pressure and the marker pressure are increased from that of the medium, so that even though they are at a higher pressure than that of the pressurization medium, the error in measuring or calibrating the pressure is reduced. On the other hand if the pressurization medium itself is the sample, such as hydrogen, then the pressure indicated by a ruby chip would be higher than that of the hydrogen. As a general conclusion, we have identified several sources of systematic error, typically of the order of up to $10 \%$, in pressure determination in DACs. When an accurate determination of the pressure is needed, these sources need to be taken into account. Some can be straightforwardly corrected for (as the position in the cell), others are more difficult to correct as they require, for example, self-consistent determination of pressure-dependent elastic constants.

\section{ACKNOWLEDGMENTS}

This research was supported by the National Science Foundation (NSF), grant DMR-0804378, the Department of Energy (DOE), grant DE-FG52-10NA29656, by the Fund for Scientific Research - Flanders, FWO projects G.0365.08 and G.0370.09N, and by the WOG WO.033.09N in Belgium. 
${ }^{1}$ A. Dewaele, P. Loubeyre, and M. Mezouar, Phys. Rev. B 70, 094112 (2004).

${ }^{2}$ A. Chijioke, W. J. Nellis, A. Soldatov, and I. F. Silvera, J. Appl. Phys. 98, 114905 (2005).

${ }^{3}$ K. Asuami and A. L. Ruoff, Phys. Rev. B 33, 5633 (1986).

${ }^{4}$ C. S. Zha, H. K. Mao, and R. J. Hemley, Phys. Rev. B. 70, 174107 (2004).

${ }^{5}$ M. Hanfland and K. Syassen, J. Appl. Phys. 57, 2752 (1985).

${ }^{6}$ H. Boppart, J. van Straaten, and I. F. Silvera, Phys. Rev. B 32, 1423 (1985).

${ }^{7}$ M. I. Eremets, J. Raman Spectrosc. 34, 515 (2003).

${ }^{8}$ M. Popov, J. Appl. Phys. 95, 5509 (2004).

${ }^{9}$ L. Sun, A. L. Ruoff, and G. Stupian, Appl. Phys. Lett. 86, 014103 (2005).

${ }^{10}$ Y. Akahama and H. Kawamura, J. Appl. Phys. 96, 3748 (2004).

${ }^{11}$ B. J. Baer, M. E. Chang, and W. J. Evans, J. Appl. Phys. 104, 034504 (2008).

${ }^{12}$ N. Dubrovinskaia, L. Dubrovinsky, R. Caracas, and M. Hanfland, Appl. Phys. Lett. 97, 251903 (2010).

${ }^{13}$ H. K. Mao, J. Xu, and P. M. Bell, J. Geophys. Res. 91, 4673 (1986).
${ }^{14}$ C. Meade and R. Jeanloz, Phys. Rev. B 42, 2532 (1990).

${ }^{15}$ K. Takemura, J. Appl. Phys 89, 662 (2001).

${ }^{16}$ K. S. Chan, T. L. Huang, T. A. Grzybowski, T. J. Whetten, and L. H. Ruoff, J. Appl. Phys 53, 6607 (1982).

${ }^{17}$ C. Bercegeay and S. Bernard, Phys. Rev. B 72, 214101 (2005).

${ }^{18}$ R. Jeanloz, B. K. Godwal, and C. Meade, Nature 349 (1991).

${ }^{19}$ H. K. Mao, Y. Wu, L. C. Chen, J. F. Shu, and A. P. Jephcoat, J. Geophys Res. 95 (1990).

${ }^{20}$ G. Steinle-Neumann, L. Stixrude, and R. Cohen, Phys. Rev. B 60, 791 (1999).

${ }^{21}$ W. Duan, B. B. Karki, and R. M. Wentzcovitch, Am. Mineral. 841961 (1999).

${ }^{22}$ F. Occelli, P. Loubeyre, and R. LeToullec, Nature Mater. 2, 151 (2003).

${ }^{23}$ R. Vogelgesang, A. K. Ramdas, S. Rodriguez, M. Grimsditch, and T. R. Anthoy, Phys. Rev. B 54, 3989 (1996).

${ }^{24}$ H. Shimizu, H. Tashiro, T. Kume, and S. Sasaki, Phys. Rev. Lett. 86, 4568 (2001). 